\title{
Mycobacterium abscessus isolated from municipal water - a potential source of human infection
}

\author{
Rachel Thomson ${ }^{1 *}$, Carla Tolson ${ }^{2}$, Hanna Sidjabat ${ }^{3}$, Flavia Huygens ${ }^{4}$ and Megan Hargreaves ${ }^{5}$
}

\begin{abstract}
Background: Mycobacterium abscessus is a rapidly growing mycobacterium responsible for progressive pulmonary disease, soft tissue and wound infections. The incidence of disease due to M. abscessus has been increasing in Queensland. In a study of Brisbane drinking water, M. abscessus was isolated from ten different locations. The aim of this study was to compare genotypically the M. abscessus isolates obtained from water to those obtained from human clinical specimens.

Methods: Between 2007 and 2009, eleven isolates confirmed as M. abscessus were recovered from potable water, one strain was isolated from a rainwater tank and another from a swimming pool and two from domestic taps. Seventy-four clinical isolates referred during the same time period were available for comparison using rep-PCR strain typing (Diversilab).

Results: The drinking water isolates formed two clusters with $\geq 97 \%$ genetic similarity (Water patterns 1 and 2). The tankwater isolate (WP4), one municipal water isolate (WP3) and the pool isolate (WP5) were distinctly different. Patient isolates formed clusters with all of the water isolates except for WP3. Further patient isolates were unrelated to the water isolates.

Conclusion: The high degree of similarity between strains of M. abscessus from potable water and strains causing infection in humans from the same geographical area, strengthens the possibility that drinking water may be the source of infection in these patients.
\end{abstract}

\section{Background}

Nontuberculous mycobacteria are environmental organisms that can cause progressive lung disease in susceptible patients. M. abscessus is a significant problem as it is highly resistant to antimicrobial agents and usually requires prolonged treatment (>six months) with intravenous and oral antibiotics in combination [1-3]. The infection is often relentless despite treatment, and even if treatment is apparently effective, relapse is common. Rapidly growing mycobacteria such as $M$. abscessus are also well documented causes of skin and soft tissue infections, especially complicating surgical procedures and injection sites [4].

Water is an important environmental reservoir of mycobacteria causing human disease [5-7]. Humans are exposed to waterborne mycobacteria through drinking,

\footnotetext{
* Correspondence: R.Thomson@uq.edu.au

${ }^{1}$ Gallipoli Medical Research Centre, Greenslopes Private Hospital, Brisbane, QLD, Australia

Full list of author information is available at the end of the article
}

swimming and bathing. Also aerosols generated during these activities may be inhaled, [8] potentially resulting in disease. There is a recognized association between pulmonary infection with rapid growing mycobacteria [9,10] (M. abscessus and M. fortuitum) and esophageal disorders. It is possible that patients acquire infection by aspirating contaminated water [11]. Outbreak investigations have identified $M$. abscessus in hospital water, dialysis [12,13] and surgical equipment and endoscopy cleaning equipment $[14,15]$. In many of these instances it is not clear whether the source of these outbreaks was an infected patient or water.

The taxonomy of Mycobacterium abscessus has been a topic of debate in the literature. M. massiliense and $M$. bolletii cannot be separated from $M$. abscessus on the basis of extensive phenotypic analysis and genotypic studies have lead to the proposition that the three taxa represent a single species with internal variability [16]. Strain variation amongst species of mycobacteria is well known and Pulsed Field Gel Electrophoresis (PFGE) has 
been considered the "Gold standard" for strain typing of many mycobacteria. ERIC (enterobacterial repetitive intergenic consensus) PCR has been used successfully to differentiate strains of mycobacteria associated with outbreaks of disease in mesotherapy clinics due to $M$. abscessus and $M$. chelonae, and in post mammoplasty patients with $M$. fortuitum infections [17,18]. A high concordance with PFGE was shown. More recently repPCR (Diversilab) was used in comparison to PFGE in a suspected outbreak of $M$. abscessus in a cystic fibrosis clinic [19]. Isolates that were identical on PFGE shared only $90 \%$ similarity with rep-PCR, suggesting that the latter method may be more discriminatory. Rep-PCR was similarly shown to concur with strain typing using VNTR (variable number tandem repeats) [20]. This method has significant advantages in cost and time saving over other methods and has demonstrated high discriminatory power.

In Queensland NTM disease remains notifiable and a central reference laboratory performs speciation of all culture positive isolates, providing a unique opportunity to capture isolates from around the state. The incidence of disease due to NTM has been increasing [21] and between 2007-2008 water sampling was conducted to investigate the presence of pathogenic NTM in potable water [22]. This paper reports the comparison of these water isolates with human isolates collected from patients in South east Queensland during the same time period.

\section{Methods}

Water isolates obtained in previous studies [22] had been stored in Dubos broth at $-20^{\circ} \mathrm{C}$ and were thawed and subbed onto $7 \mathrm{H} 11$ plates as well as Lowenstein-Jensen slopes and incubated at $35^{\circ} \mathrm{C}$ until sufficient growth was available.

Those identified using $16 \mathrm{~S}$ rDNA sequencing as $M$. abscessus/M. chelonae underwent hsp 65 and rpoB gene fragment sequencing for more definitive identification. According to the proposed changes by Leao et al. [16] in line with the Bacteriological code, for the purposes of this study all isolates identified as $M$. abscessus, $M$. bolletii, M. massiliense were included as $M$. abscessus species.

Human samples that were collected as part of routine patient care, were digested and decontaminated using $4 \%$ $\mathrm{NaOH}$, neutralized with phosphoric acid and centrifuged at $3000 \mathrm{~g}$ to concentrate the acid-fast bacilli (AFB). Smears were prepared from the sediment and stained by the Ziehl-Niehlsen (ZN) method. One Lowenstein-Jensen slope ( \pm pyruvate) and $7 \mathrm{ml}$ Mycobacterial Growth Indicator Tube (MGIT) were inoculated and incubated at $35^{\circ} \mathrm{C}$ until growth was detected. $\mathrm{ZN}$ staining of colonies confirmed AFB. Multiplex PCR was performed to discriminate between $M$. tuberculosis, M. avium, M. intracellulare, $M$. abscessus and other Mycobacterium spp. Isolates identified as other Mycobacterium spp were further speciated using Hain Life Sciences GenoType ${ }^{\circ}$ Mycobacterium CM kit (2004-7 only) and/or 16S rDNA sequencing in conjunction with phenotypic characteristics. All clinical isolates underwent gene fragment sequencing for $h s p 65$ and rpoB.

\section{Strain typing using automated Rep-PCR}

The clonality of clinical and water $M$. abscessus isolates and a control strain (ATCC 19977) was determined using a rep-PCR based method (Diversilab ${ }^{\circ}$ system, bioMerieux, Melbourne). DNA was extracted from clinical and water isolates using the Ultraclean Microbial DNA Isolation Kit (Mo Bio Laboratories, CA, USA). PCR mixture was prepared using AmpliTaq polymerase and PCR buffer (Applied Biosystems, New Jersey, USA) and Mycobacterium Diversilab $^{\circ}$ primer mix according to the manufacturer's instructions. Separation and detection of Rep-PCR products was performed by micro-fluidic chips of the Diversilab ${ }^{\circ}$ System. A laboratory control strain of M. abscessus was included, and two chips were repeated because of low intensity banding. In most cases when the intensity improved the pattern didn't change from that obtained with low intensity. An additional chip was run twice to confirm reproducibility of strain patterns. Any aberrances were excluded from the analysis. Fingerprints were analysed with Diversilab ${ }^{\circ}$ software v.3.4.38 using the Pearson correlation co-efficient and unweighted pair group method with arithmetic means to compare isolates and determine clonal relationship. A similarity index of $\geq 97 \%$ was used to define isolates that were indistinguishable, based on the manufacturers recommendations despite previously published comparisons of rep-PCR with both PFGE and Variable Number Tandem Repeats, [20] where a cut off of $90 \%$ was used. Isolates that shared $95 \%$ similarity were considered similar (and formed groups), and those $<90 \%$ considered 'different/unrelated'.

The study protocol was approved by the Human Research Ethics Committee of The Prince Charles Hospital (EC-2617).

\section{Results}

During the 10 years from 2001-2010, there were 486 patient notifications: $68.1 \%$ were pulmonary, $22.2 \%$ cutaneous or soft tissue (Table 1).

Seventy-four clinical isolates collected during 2007-8 were available for comparison with fifteen water isolates. The mean age of patients was 55.94 yrs (SD 22.25; median 58.5; range 2-90yrs). Fifty percent were male. Fortyfour patients had clinically significant pulmonary disease (according to clinician notification and the ATS/IDSA criteria), 11 patients had pulmonary isolates that were 
Table 1 Sites of isolation of $M$. abscessus from human samples 2001-2010

\begin{tabular}{lc}
\hline Site of isolate & Frequency (\%) \\
\hline Blood & $14(2.9)$ \\
Bones and joints & $5(1)$ \\
Cutaneous/Soft tissue & $108(22.2)$ \\
Eye & $1(0.2)$ \\
Lymph node + Other & $1(0.2)$ \\
Lymph nodes & $3(0.6)$ \\
Peritoneal & $2(0.4)$ \\
Post surgical/Medical access device & $19(3.9)$ \\
Pulmonary & $331(68.1)$ \\
Unknown & $2(0.4)$ \\
Total & $486(100)$ \\
\hline
\end{tabular}

considered contaminants or not clinically significant and 19 patients had isolates from extra-pulmonary sites.

Eleven patients (14.9\%) were included from other parts of Australia who lived $>500 \mathrm{~km}$ outside the Brisbane water distribution network, and would have received potable water supply to their homes from different networks. Of the remaining patients, 31/63 (49.2\%) patients lived in reservoir zones that contained sample points that grew M. abscessus.
Of 19 water isolates identified as M. abscessus/ M. chelonae using 16s rDNA sequencing, 17 underwent successful hsp65 and rpoB sequencing. This identified 14 isolates as $M$. abscessus and one as M. bolletii - all considered $M$. abscessus subs abscessus according to the proposed taxonomy by Leao [16]. There was insufficient DNA in two samples. Fifteen water isolates of $M$. abscessus were strain typed using rep-PCR. Eleven isolates came from six different potable water distribution system sampling sites; two came from domestic taps in the same dwelling, one came from an unrelated rainwater tank and the other from a suburban domestic swimming pool.

The 15 water isolates formed 6 different patterns (Figure 1). Municipal water isolates formed two clusters of indistinguishable isolates ( $\geq 97 \%$ similarity) and were classified as Group WP1 (eight isolates) and Group WP2 (two isolates) and a single isolate WP3. The rainwater tank isolate (Group WP4) and the swimming pool isolate (Group WP5) were distinctly different. The two isolates from a patient's domestic taps were indistinguishable (WP 6) (Figure 1).

When the clinical isolates were analysed with the water isolates there were 28 patterns/clusters that shared $>97 \%$ similarity (indistinguishable). Thirteen of these clusters contained more than 1 isolate, and the remaining patterns were single isolates only. The indistinguishable
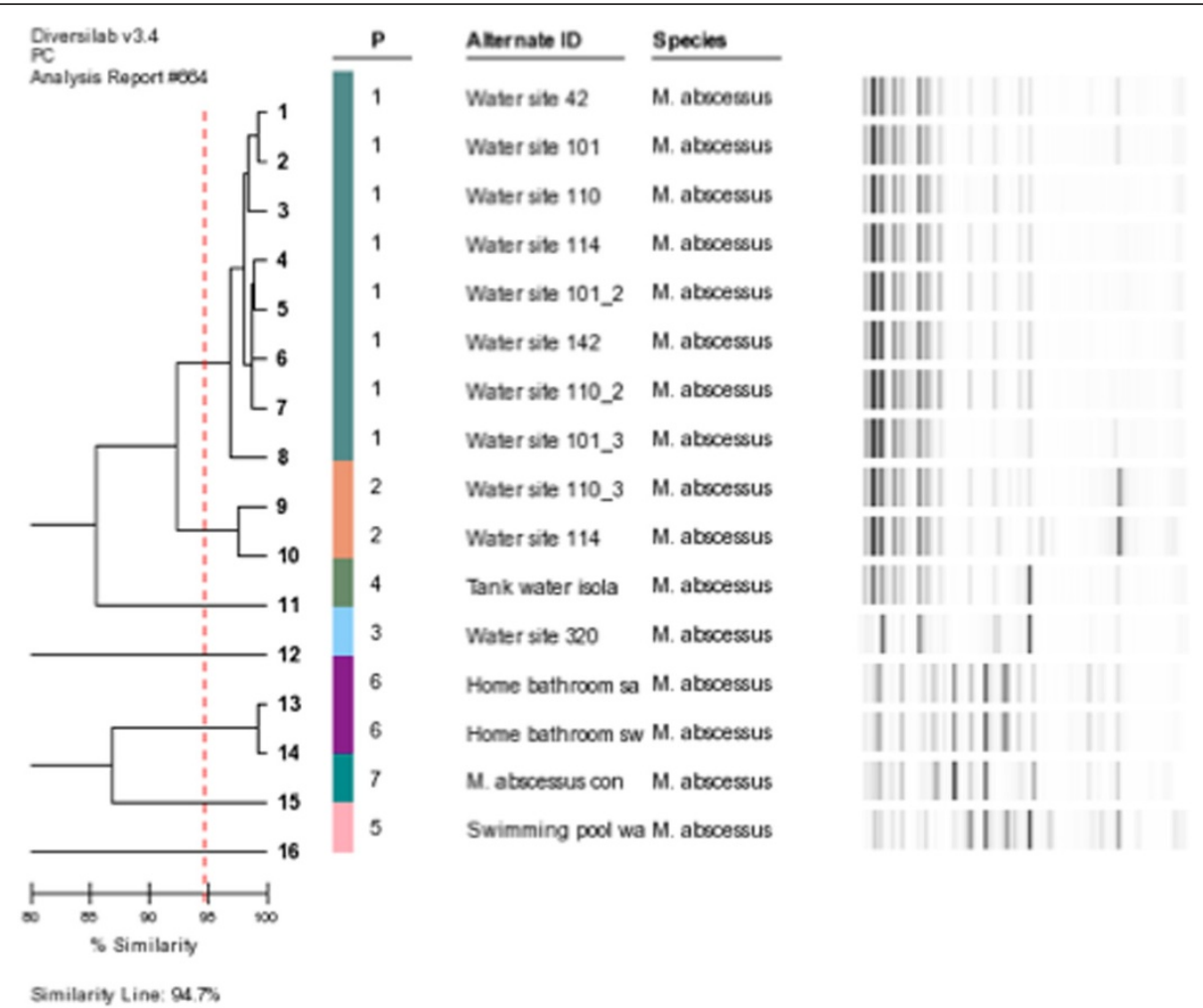

Figure 1 rep-PCR dendrogram of $M$. abscessus water strains with Pearson Correlation Analysis. 
clusters that were similar ( $>95 \%$ similarity) were then grouped into seven main groups. Full report and graphics in Additional file 1.

There were two large similar clusters of 21 (P15) and 22 (P17) isolates respectively. Examples demonstrated in Figure 2. P15 included one of the municipal water isolates from WP1 and P17 contained the remaining seven isolates from WP1. A further three clusters (P1214) containing 5 clinical isolates were similar to cluster P15 and a single isolate P16 was similar to P17.

The two water isolates from WP2 also clustered with six clinical isolates (P20-22;Gp 4: Additional file 1). Five clinical isolates were indistinguishable from the tank water isolate (P2). A further three clinical isolates and the two domestic tap water isolates were similar to this cluster (Group 1). One clinical isolate was indistinguishable from the swimming pool isolate Figure 3.
The isolate from water site 320 (WP3) differed from all the clinical isolates.

There were no significant differences between the characteristics of groups of patients in each strain cluster (age, gender, type of disease, site of infection, ground water source or reservoir zone) (Table 2). Some of the isolates from more remote areas did have unique strain patterns, but equally so did some of the Brisbane area isolates. Some of the remote area isolates were similar to those of patients from Brisbane. However as is often the case with NTM infections, the precise location of where infection is acquired can be difficult to pinpoint.

\section{Discussion}

M. abscessus has become an increasingly important clinical problem in the last 10 years, and its presence in potable water has not previously been emphasized.

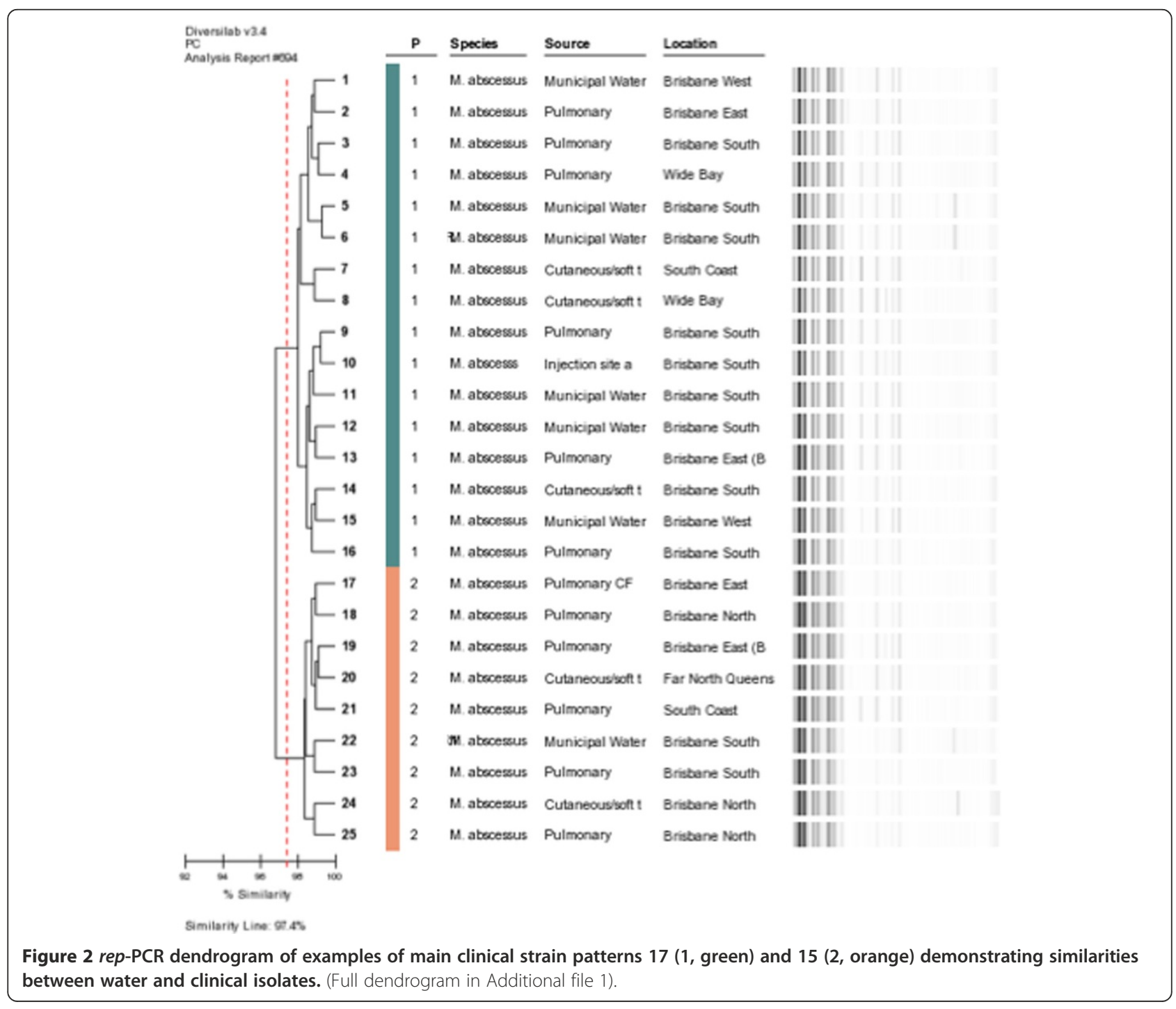




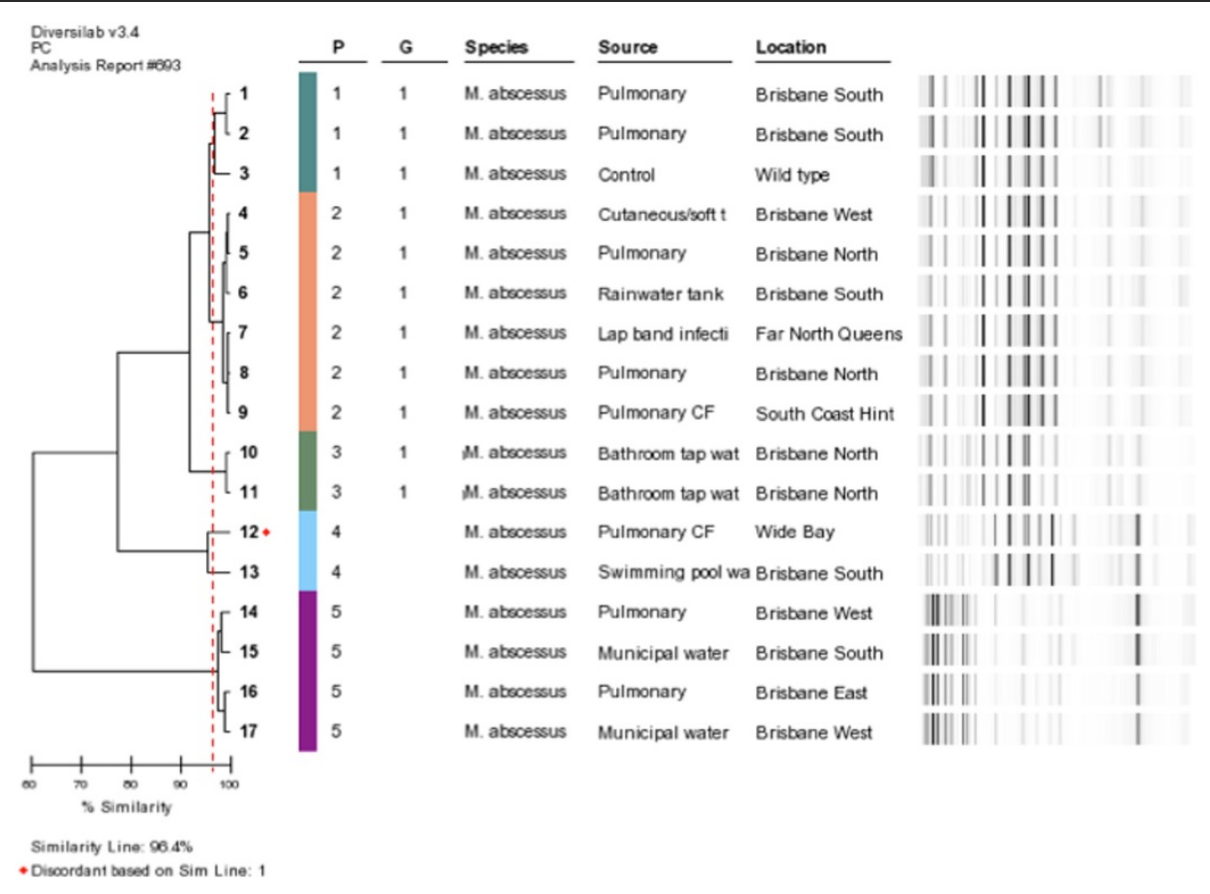

Figure 3 rep-PCR dendrogram showing examples of different strain patterns where there were similarities between clinical and water strains.

In the majority of published studies looking for NTM in water, no $M$. abscessus was documented [23]. However there have been taxonomical changes that have led to $M$. abscessus being recognized as independent from $M$. chelonae, so older studies reporting $M$. chelonae may not necessarily have differentiated $M$. chelonae subsp abscessus, particularly as both species have identical $16 \mathrm{~s}$ rDNA sequences. But in studies of potable water done since 2000 M. abscessus has been rarely reported.

A study done in Pretoria, South Africa in 2004 [24] reported an analysis of 78 biofilm and water samples -14 had NTM - no MAC, one had M. abscessus (16s rDNA sequencing only). In a multi-hospital outbreak investigation in Taiwan, M. abscessus was grown from one water sample from one of the hospitals involved however this differed on strain typing from the isolates found in patients [25]. Falkinham [26] in 2001 sampled 8 different water treatment systems (raw, treated and Distribution System (DS) samples). M. abscessus was only found in one raw water sample from one system. There were no rapid growers in treated water, two rapid growers were found in the DS samples but the paper doesn't clarify the species. In a more recent study (2009), van Ingen reported no M. abscessus in shower or tap water in the Netherlands [27]. We have found that the inclusion of liquid media increased the yield for M. abscessus from potable water samples [22]. However

Table 2 Clinical details of patients with M. abscessus isolates used for strain typing comparison

\begin{tabular}{|c|c|c|c|c|}
\hline & n (\%) & Age* Mean \pm SD & Gender M:F & Species \\
\hline \multirow[t]{2}{*}{ Lung } & $54(73)$ & $58.24 \pm 24.53$ & $27: 27$ & M. abscessus subs abscessus 34 (63\%) \\
\hline & & & & M. abscessus subs bolletii 20 (37\%) \\
\hline \multirow[t]{2}{*}{ Cutaneous/Soft Tissue Infection } & $11(14.9)$ & $49.18 \pm 14.49$ & $5: 6$ & M. abscessus subs abscessus 9 (81.8\%) \\
\hline & & & & M. abscessus subs bolletii 2 (18.2\%) \\
\hline Invasive Device/Line & $3(4.1)$ & $42.67 \pm 19.63$ & $2: 1$ & M. abscessus subs abscessus 3 (100\%) \\
\hline \multirow[t]{2}{*}{ Surgical Wound Infection } & $2(2.7)$ & $46.00 \pm 7.07$ & $1: 1$ & M. abscessus subs abscessus 1 (50\%) \\
\hline & & & & M. abscessus subs bolletii 1 (50\%) \\
\hline Bloodstream Infection (Line Assoc) & $1(1.4)$ & $47.00 \pm 19.86$ & $1: 0$ & M. abscessus subs abscessus 1 (100\%) \\
\hline Injection Site & $3(4.1)$ & $41.33 \pm 22.75$ & $2: 1$ & M. abscessus subs abscessus 3 (100\%) \\
\hline \multirow[t]{2}{*}{ Total } & $74(100)$ & $55.09 \pm 22.88$ & $38: 36$ & M. abscessus subs abscessus 51 (68.9\%) \\
\hline & & & & M. abscessus subs bolletii 23 (31.1\%) \\
\hline
\end{tabular}


three of the 13 isolates were only grown on solid media suggesting that for future studies using culture-based techniques, both media forms should be included.

It has long been recognized that outbreaks of disease due to rapid growers may have originated in hospital and tap water, particularly for $M$. chelonae and $M$. fortuitum [28] though in some studies the authors have acknowledged that speciation separating $M$. abscessus has not occurred. Outbreak investigations have linked $M$. abscessus infections and pseudo infections to hospital tap water, and endoscope cleaning fluids $[29,30]$.

In all of these hospital outbreaks, the potential for contamination of hospital sources and equipment by infected patients or other environmental sources (such as dust or dirt) exists; hence the origin of the culprit strain remains unclear.

Zelazny [31] compared partial sequencing of rpoB, hsp 65 and secA gene fragments and rep-PCR (Diversilab) in order to differentiate $M$. abscessus from subspecies formerly named $M$. massiliense and M. bolletii. They were able to group the majority of $M$. abscessus complex clinical isolates into a group similar to the ATCC strain 19977 , with a degree of diversity as low as $80 \%$ similarity to the reference strain. There were two other strain groups neither of which resembled the strain patterns obtained in our study. The geographic origin of the patients included in this study was not reported nor examined, but as the study was performed by a tertiary referral centre many came from patients around the United States (A. Zelazny, personal communication). In our study the strains obtained both from patients and from water were closely related, and differed from the strain patterns reported in the US, suggesting geographical differences in strain variation. The diversity of the US strains, may be due to the diverse origins of the isolates reported, reflecting differences in the dominant environmental strains. There was a degree of geographic diversity of our strains with unique strain types identified from central and far north Queensland $(>500 \mathrm{~km}$ and $>1000 \mathrm{~km}$ from Brisbane respectively) and two unique strains from the bayside of Brisbane (> 30km from the CBD).

Whilst rep-PCR using the Diversilab has only recently been used for strain typing mycobacteria, published studies support its performance as equivalent or perhaps even more discriminatory than PFGE. Aitken et al [19] reported an outbreak of $M$. abscessus infections in a lung transplantation and cystic fibrosis center. Isolates involved were strain typed using both PFGE and rep-PCR (Diversilab). The isolate of the index case and outbreak cases were reported to be "genetically identical" using PFGE. The repPCR patterns of the same isolates showed only $90 \%$ genetic similarity, suggesting in fact that these strains are different, and that rep-PCR may be more discriminatory than PFGE.
Harris [20] describes a novel VNTR (variable number tandem repeats) method to type $41 \mathrm{M}$. abscessus complex strains from 17 pediatric cystic fibrosis patients and compares this with rep-PCR (Diversilab). As with the Aitken paper, isolates with $90 \%$ similarity were regarded as indistinguishable. The finding of six distinct profiles among 12 patients with $M$. abscessus complex suggests that the typing methods have sufficient discriminatory power.

\section{Conclusion}

We have analysed both patient and water isolates from quite diverse and epidemiologically unrelated settings across a geographical area of $>1367 \mathrm{~km}^{2}$ and found 28 clusters. We have more definitively identified $M$. abscessus strains in potable water, as strains that are indistinguishable from those found in samples from patients known to have disease. Whilst point source investigations of outbreaks have linked $M$. abscessus infection and hospital water and equipment, the present study tightens the link between potable water, to which patients are regularly exposed, and the acquisition of infection. This has important implications for those patients at risk. Given the difficulty in treating these infections and the devastating effects they have for patients, efforts to identify effective disinfection methods for M. abscessus should be a high priority.

\section{Additional file}

Additional file 1: Full Diversilab rep-PCR analysis of water and human isolates.

Competing interests

All authors declare that they have no competing interests.

Authors' contributions

RT conceived and designed the study, processed the water samples, performed the Diversilab analysis and wrote the manuscript. CT processed water and human samples, performed DNA extraction and rep-PCR. HS performed rep-PCR experiments with $\mathrm{CT}$. FH and $\mathrm{MH}$ contributed to the design and conduct of the study, the analysis of the results, and the writing of the manuscript. All authors reviewed and approved the final manuscript.

\section{Acknowledgements}

This work was supported by research grants from The Gallipoli Medical Research Foundation and The Prince Charles Hospital Foundation, Brisbane, Australia. The authors acknowledge the contribution of Dr Chris Coulter and staff at the QLD Mycobacterial Reference Laboratory for their support.

\section{Author details}

${ }^{1}$ Gallipoli Medical Research Centre, Greenslopes Private Hospital, Brisbane, QLD, Australia. ${ }^{2}$ QLD Mycobacterial Reference Laboratory, Pathology Queensland, RBWH Campus, Herston Rd, Herston, QLD 4006, Australia. ${ }^{3}$ University of Queensland Centre for Clinical Research, Herston Rd, Herston, QLD 4006, Australia. ${ }^{4}$ Institute of Health and Biomedical Innovation, Kelvin Grove Campus, Queensland University of Technology, Brisbane, QLD 4059, Australia. ${ }^{5}$ Queensland University of Technology, Faculty of Science and Technology, George Street, Brisbane, QLD 4001, Australia.

Received: 29 January 2013 Accepted: 10 May 2013

Published: 25 May 2013 


\section{References}

1. Jeon K, Kwon OJ, Lee NY, Kim B-J, Kook Y-H, Lee S-H, Park YK, Kim CK, Koh W-J: Antibiotic treatment of Mycobacterium abscessus lung disease: a retrospective analysis of 65 patients. Am J Respir Crit Care Med 2009, 180(9):896-902.

2. Thomson R, Yew W-W: When and how to treat pulmonary non-tuberculous mycobacterial diseases. Respirology 2009, 14(1):12-26.

3. Griffith DE, Aksamit T, Brown-Elliott BA, Catanzaro A, Daley C, Gordin F, Holland SM, Horsburgh R, Huitt G, lademarco MF, Iseman M, Olivier K, Ruoss S, von Reyn CF, Wallace RJ Jr. Winthrop K, on behalf of the ATS Mycobacterial Diseases Subcommitte: An Official ATS/IDSA statement: diagnosis, treatment, and prevention of nontuberculous mycobacterial diseases. Am J Respir Crit Care Med 2007, 175:367-416.

4. De Groote MA, Huitt G: Infections due to rapidly growing Mycobacteria. Clin Infect Dis 2006, 42(12):1756-63.

5. Covert TC, Rodgers MR, Reyes AL, Stelma GN Jr: Occurrence of nontuberculous mycobacteria in environmental samples. Appl Environ Microbiol 1999, 65(6):2492-6.

6. Fox C, Smith B, Brogan O, Rayner A, Harris G, Watt B: Non-tuberculous mycobacteria in a hospital's piped water supply. J Hosp Infect 1992, 21(2):152-4.

7. Le Dantec C, Duguet J-P, Montiel A, Dumoutier N, Dubrou S, Vincent V: Occurrence of mycobacteria in water treatment lines and in water distribution systems. App/ Environ Microbiol 2002, 68(11):5318-25.

8. Falkinham J III: Mycobacterial aerosols and respiratory disease. Emerg Infect Dis. 2003, 9:763-7.

9. Hadjiliadis D, Adlakha A, Prakash UB: Rapidly growing mycobacterial lung infection in association with esophageal disorders. Mayo Clinic Proc. 1999, 74:45-51.

10. Hutchins GM, Boitnott JK: Atypical mycobacterial infection complicating mineral oil pneumonia. JAMA. 1978, 240:539-41.

11. Thomson R, Armstrong J, Looke D: Gastroesophageal reflux disease, acid suppression, and Mycobacterium avium complex pulmonary disease. Chest 2007, 131:1166-72

12. Carson LA, Bland LA, Cusick LB, Favero MS, Bolan GA, Reingold AL, Good RC: Prevalence of nontuberculous mycobacteria in water supplies of hemodialysis centers. Appl Environ Microbiol 1988, 54(12):3122-5.

13. Gomila M, Ramirez A, Lalucat J: Diversity of environmental mycobacterium isolates from hemodialysis water as shown by a multigene sequencing approach. Appl Environ Microbiol 2007, 73(12):3787-97.

14. Gubler J, Salfinger M, von Graevenitz A: Pseudoepidemic of nontuberculous mycobacteria due to a contaminated bronchoscope cleaning machine. Report of an outbreak and review of the literature. Chest 1992, 101(5):1245-9.

15. Dawson DJ, Armstrong J, Blacklock ZM: Mycobacterial cross-contamination of bronchoscopy specimens. Am Rev Respir Dis 1982, 126(6):1095-7.

16. Leao SC, Tortoli E, Euzeby JP, Garcia MJ: Proposal that Mycobacterium massiliense and Mycobacterium bolletii be united and reclassified as Mycobacterium abscessus subsp. bolletii comb. nov., designation of Mycobacterium abscessus subsp. abscessus subsp. nov. and emended description of Mycobacterium abscessus. Int J Syst Evol Microbiol. 2011, 61:2311-3.

17. Sampaio JLM, Viana-Niero C, de Freitas D, Hfling-Lima AL, Leao SC Enterobacterial repetitive intergenic consensus PCR is a useful tool for typing Mycobacterium chelonae and Mycobacterium abscessus isolates. Diagn Micr Infec Dis 2006, 55(2):107-18.

18. Sampaio JLM, Chimara E, Ferrazoli L, da Silva Telles MA, del Guercio VMF, Jerico ZVN, Miyashiro K, Fortaleza CMCB, Padoveze MC, Leao SC: Application of four molecular typing methods for analysis of Mycobacterium fortuitum group strains causing post-mammaplasty infections. Clin Microbiol Inf 2006, 12(2):142-9.

19. Aitken ML, Limaye A, Pottinger P, Whimbey E, Goss CH, Tonelli MR, Cangelosi GA, Dirac MA, Olivier KN, Brown-Elliott BA, McNulty S, Wallace RJ: Respiratory outbreak of Mycobacterium abscessus subspecies massiliense in a lung transplant and cystic fibrosis center. Am J Respir Crit Care Med 2012, 185(2):231-2

20. Harris KA, Kenna DTD, Blauwendraat C, Hartley JC, Turton JF, Aurora P, Dixon GLJ: Molecular fingerprinting of Mycobacterium abscessus strains in a cohort of pediatric cystic fibrosis patients. J Clin Microbiol, 50(5):1758-61.

21. Thomson R: Changing epidemiology of pulmonary nontuberculous mycobacteria infections. Emerg Inf Diseases 2010, 16(10):1576-82.
22. Thomson RM, Carter R, Tolson C, Coulter C, Huygens F, Hargreaves M: Factors associated with the isolation of Nontuberculous Mycobacteria (NTM) from a large municipal water system in Brisbane, Australia. BMC Microbiology, 13:89.

23. Van Ingen J, Boeree M, Dekhuijzen P, Van Soolingen D: Environmental sources of rapid growing nontuberculous mycobacteria causing disease in humans. Clin Micro Inf 2009, 15:888-892.

24. September SM, Brozel VS, Venter SN: Diversity of nontuberculoid Mycobacterium species in biofilms of urban and semiurban drinking water distribution systems. App/ Environ Microbiol 2004, 70(12):7571-3.

25. Huang W-C, Chiou C-S, Chen J-H, Shen G-H: Molecular epidemiology of Mycobacterium abscessus infections in a subtropical chronic ventilatory setting. J Med Microbiol 2010, 59(10):1203-11.

26. Falkinham J III, Norton C, Le Chavallier M: Factors influencing numbers of Mycobacterium avium, Mycobacterium intracellulare and other mycobacteria in drinking water distribution systems. Appl Environ Microbiol 2001, 67(3):1225-31.

27. Van Ingen J, Blaak H, de Beer J, de Roda Husman AM, Van Soolingen D: Rapidly growing nontuberculous mycobacteria cultured from home tap and shower water. App Environ Microbiol 2010, 76(17):6017-6019.

28. Wallace RJ, Brown BA, Griffith DE: Nosocomial Outbreaks/pseudooutbreaks caused by nontuberculous mycobacteria. Annu Rev Microbiol 1998, 52(1):453-90.

29. Maloney S, Welbel S, Daves B, Adams K, Becker S, Bland L, Arduino M, Wallace R Jr, Zhang Y, Buck G, Risch P, Jarvis WR: Mycobacterium abscessus pseudoinfection traced to an automated endoscope washer: utility of epidemiologic and laboratory investigation. J Infect Dis 1994, 169:1166-9.

30. Zhang Y, Rajagopalan M, Brown BA, Wallace RJ Jr: Randomly amplified polymorphic DNA PCR for comparison of Mycobacterium abscessus strains from nosocomial outbreaks. J Clin Microbiol 1997, 35(12):3132-9.

31. Zelazny AM, Root JM, Shea YR, Colombo RE, Shamputa IC, Stock F, Conlan S, McNulty S, Brown-Elliott BA, Wallace RJ Jr, Olivier KN, Holland SM, Sampaio EP: Cohort study of molecular identification and typing of Mycobacterium abscessus, Mycobacterium massiliense, and Mycobacterium bolletii. J Clin Microbiol 2009, 47(7):1985-95.

doi:10.1186/1471-2334-13-241

Cite this article as: Thomson et al:: Mycobacterium abscessus isolated from municipal water - a potential source of human infection. BMC Infectious Diseases 2013 13:241.

\section{Submit your next manuscript to BioMed Central and take full advantage of:}

- Convenient online submission

- Thorough peer review

- No space constraints or color figure charges

- Immediate publication on acceptance

- Inclusion in PubMed, CAS, Scopus and Google Scholar

- Research which is freely available for redistribution
C) BioMed Central 